\title{
Cosmic ray energy spectrum in the 2nd knee region measured by the TALE-SD array
}

\author{
Koki Sato $^{a, *}$ on behalf of the Telescope Array Collaboration ${ }^{\dagger}$ \\ ${ }^{a}$ Graduate School of Science, Osaka City University, Osaka, Japan \\ E-mail: m20sa019@vt.osaka-cu.ac.jp
}

The Telescope Array Low energy Extension (TALE) experiment in Utah, U.S.A., consists of 10 atmospheric fluorescence telescopes and 80 Surface Detectors (SDs) spread over an area of $21 \mathrm{~km}^{2}$. The SD array consists of $40 \mathrm{SDs}$ at $400 \mathrm{~m}$ spacing and $40 \mathrm{SDs}$ at $600 \mathrm{~m}$ spacing. The TALE-SD was completed in February 2018 and has been in steady operation since then, triggering at a rate of about 30 air shower events in 10 minutes. We have developed the software to measure the energy spectrum of cosmic rays from the data obtained by TALE-SD. The performance of the software was evaluated by using air shower events generated by Monte Carlo simulation. We estimate that when the energy of the primary cosmic ray is $10^{18.0} \mathrm{eV}$, the accuracy of energy determination is $20 \%$, the accuracy of arrival direction determination is $1.5^{\circ}$, and the aperture is $16 \mathrm{~km}^{2} \mathrm{sr}$. We also compared the MC and Data to see if they were consistent. In this presentation, we will report these results.

$37^{\text {th }}$ International Cosmic Ray Conference (ICRC 2021)

July 12 th - 23rd, 2021

Online - Berlin, Germany

\footnotetext{
${ }^{*}$ Presenter

${ }^{\dagger}$ A complete list of collaborators see Pos(ICRC2021)
} 


\section{Introduction}

The Telescope Array (TA) is deployed in Millard County, Utah, USA. It mainly observes Ultra High Energy Cosmic Rays (UHECRs), using both fluorescence telescopes and scintillator surface detectors. There are 507 surface detectors with about $700 \mathrm{~km}^{2}$ effective detection area and The three TA FD stations, which are called Black Rock Mesa (BRM), Long Ridge (LR), and Middle Drum(MD). These TA FD telescopes are viewing $3^{\circ}$ to $31^{\circ}$ in elevation. A map of the detectors is shown in left Figure 1.

The Telescope Array also has an extension to lower the experiment's energy threshold allowing us to study cosmic rays with energies down below $10^{16.0} \mathrm{eV}$. This extension is known as the Telescope Array Low Energy extension (TALE) shown in right Figure 1. In the TALE experiment, 10 Fluorescence detectors, which observes at a higher elevation angle than the TA-FD, and 80 surface detectors, which is installed at a higher density than the TA-SD, are installed next to the TA experiment. The full details of the detectors are found in [1] [2] .
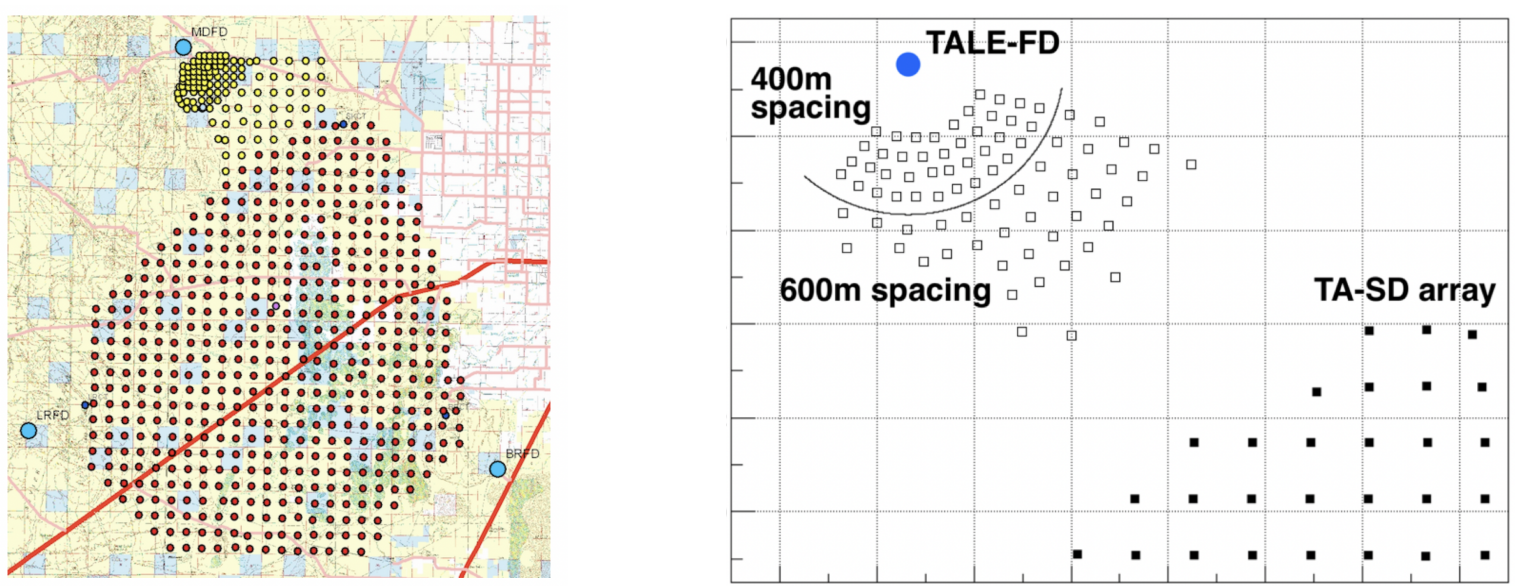

Figure 1: Map of Telescope Array and TALE detectors. Left : Locations of the scintillator Surface Detectors (SD) are shown as red points and the locations of the three Fluorescence Detector (FD) stations are indicated by the blue points. Locations of the scintillator Surface Detectors for TALE are shown as yellow points.

Right : Locations of the scintillator Surface Detectors and the TALE Fluorescence Detector (TALE-FD) stations are shown as white points and blue points respectively. Locations of the scintillator Surface Detectors for TA are shown as balck points. The area closest to the TALE-FD station have 40 SDs with a spacing of $400 \mathrm{~m}$, and the outer area have 40 SDs with a spacing of $600 \mathrm{~m}$. TALE-SD array is arranged in a fan shape instead of a grid shape in order to optimize the hybrid observation with TALE-FD.

The goal of the TALE experiment is to measure the energy spectrum and mass composition in the energy range from $10^{15.0} \mathrm{eV}$ to $10^{20.5} \mathrm{eV}$ and identify some interesting spectral features such as the second knee and even the knee. For this analysis, we focus on the 2nd knee region (primary energy is around $10^{17.5} \mathrm{eV}$ ), and utilize a reconstruction of the shower events by the TALE Surface Detectors (TALE-SD). 


\section{Event reconstruction and selection}

About 4 months of data from October 2019 to January 2020 were analyzed for the cosmic ray spectrum. Total detector on-time is $\sim 3600$ hours. In this analysis, the events are reconstructed by the equation for the geometric reconstruction of the event,

$$
t^{\mathrm{FIT}}=T_{0}+\frac{l}{c}+\tau
$$

where $t^{\mathrm{FIT}}$ is arrival time of air shower to $\mathrm{SD}, T_{0}$ is arrival time of the shower core to the surface, $\mathrm{c}$ is the speed of the light and $\tau$ is time delay in the arrival time of the particle from the shower plane due to the curvature of the shower surface. This $\tau$ is a parameterization of the equation obtained by Linsley as a function of zenith angle $\theta$ for the TA experiment[3][4] [5] . Next reconstruction step, the particle density obtained from each detector is fitted with the following lateral distribution function from Akeno Giant Air Shower Array (AGASA) experiments [6]

$$
\begin{aligned}
& \rho^{\mathrm{FIT}}=A\left(\frac{s}{91.6[m]}\right)^{-1.2}\left(1+\frac{s}{91.6[m]}\right)^{-(\eta(\theta)-1.2)}\left(1+\left(\frac{s}{1000[m]}\right)^{2}\right)^{-0.6}\left[/ m^{2}\right] \\
& \eta(\theta)=3.97-1.79(\sec \theta-1)
\end{aligned}
$$

where $\mathrm{A}$ is the normalization, $\mathrm{s}$ is the distance to the shower axis, $\theta$ is the zenith angle.

The TALE-SD uses two reconstructed parameters to determine the primary energy. One is the particle density at $600 \mathrm{~m}$ from the shower axis ( $\mathrm{S} 600)$ and the other is the zenith angle $(\theta)$. The primary energy is determined using these parameters and the energy table for TALE-SD array obtained from the air shower Monte Carlo simulation as shown Figure2. This energy estimation table assumes a pure proton, and is made using the quality cuts shown below table1. Also, to obtain good resolution and Data/Monte Carlo comparisons, quality cuts were performed on the reconstructed showers as summarized in 1 .

\begin{tabular}{ll}
\hline \hline Variable & cut condition \\
\hline \# of SDs & $\geq 5$ \\
zenith angle $\left[{ }^{\circ}\right]$ & $<45^{\circ}$ \\
$\mathrm{D}_{\text {border }}[\mathrm{m}]$ & $<100 \mathrm{~m}$ \\
$\chi_{\mathrm{G}}^{2} /$ d.o.f & $<4$ \\
$\chi_{\mathrm{L}}^{2} /$ d.o.f & $<2$ \\
$\left(\sigma_{\theta}^{2}+\sin ^{2} \theta \sigma_{\phi}^{2}\right)^{1 / 2}\left[{ }^{\circ}\right]$ & $<2.5^{\circ}$ \\
$\sigma_{\mathrm{S} 600} / \mathrm{S} 600$ & $<0.25$ \\
\hline
\end{tabular}

Table 1: Quality Cuts Applied in this study 


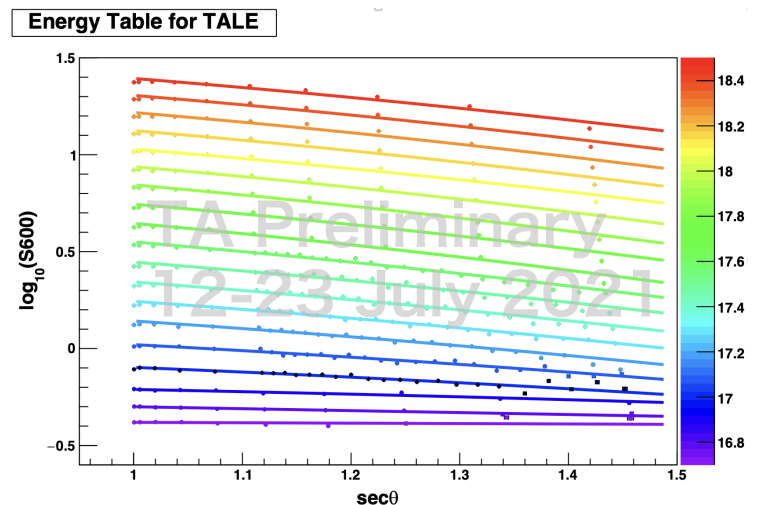

Figure 2: Energy Estimation Table for TALE-SD array. This energy estimation table assumes a pure proton. At this study, the energy range that can be estimated is from $10^{16.7} \mathrm{eV}$ to $10^{18.5} \mathrm{eV}$.

\section{Monte Carlo simulation}

We generate cosmic ray showers using the CORSIKA-based MC simulation code developed for TA [7]. The Monte Carlo simulation procedure consists of two parts. One is an air shower simulation with the hadron interaction model QGSJET-II-04 [8] , and the other is the TALE surface detector simulation. In this analysis, we assume that the primary particle is proton. These showers were thrown from $10^{16.95} \mathrm{eV}$ to $10^{18.55} \mathrm{eV}$ with the spectral index of 3.0. The Monte Carlo simulated an isotropic distribution in the azimuthal angle, and zenith angle was thrown up to $65^{\circ}$. And core position is randomly chosen within a circle of radius $5.5 \mathrm{~km}$, which is enough to cover the entire TALE-SD array.
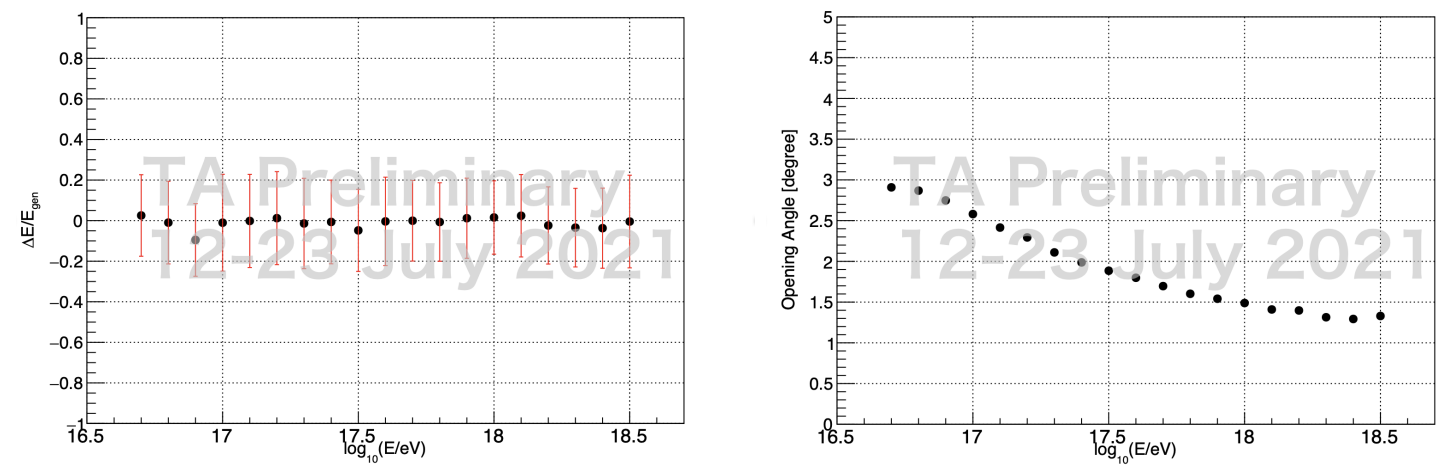

Figure 3: Resolution studies using Monte Carlo events. Left: primary energy. The Gaussian fit is used to determine the detector bias and resolution. Right: Opening angle at $68 \%$ of the number of events at a given energy.

Figure 3 show the resolution of important parameters from reconstruction. We evaluate the resolutions of $\sim 20 \%$, shown as the red bars, in primary energy range from $10^{16.7} \mathrm{eV}$ to $10^{18.5} \mathrm{eV}$, and as for the resolution of the arrival direction, $3^{\circ}$ for events with energy $10^{16.7} \mathrm{eV}$, and $1.5^{\circ}$ for events with energy $10^{18.5} \mathrm{eV}$. 
In the future, to evaluate the energy spectrum, it is necessary to calculate the aperture of the TALE-SD array. The aperture cannot be calculated with simple geometric factor because it depends on the performance of the surface detectors and also on the primary particles. Therefore, these dependencies are taken into account by MC simulation to estimate the aperture of the detector. However, note that in this analysis we assume pure proton in the MC. The aperture is calculated as follows

$$
A \Omega(E)=A_{\mathrm{GEN}} \Omega_{\mathrm{GEN}} \cdot \mathrm{N}_{\text {recon }}(\mathrm{E}) / \mathrm{N}_{\text {thrown }}(\mathrm{E})
$$

where $\mathrm{E}$ is the primary energy of cosmic ray, $A_{\mathrm{GEN}} \Omega_{\mathrm{GEN}}$ is the thrown aperture region of MC simulation, $N_{\text {recon }}$ is the number of reconstructed events and $N_{\text {thrown }}$ is the number of thrown events.

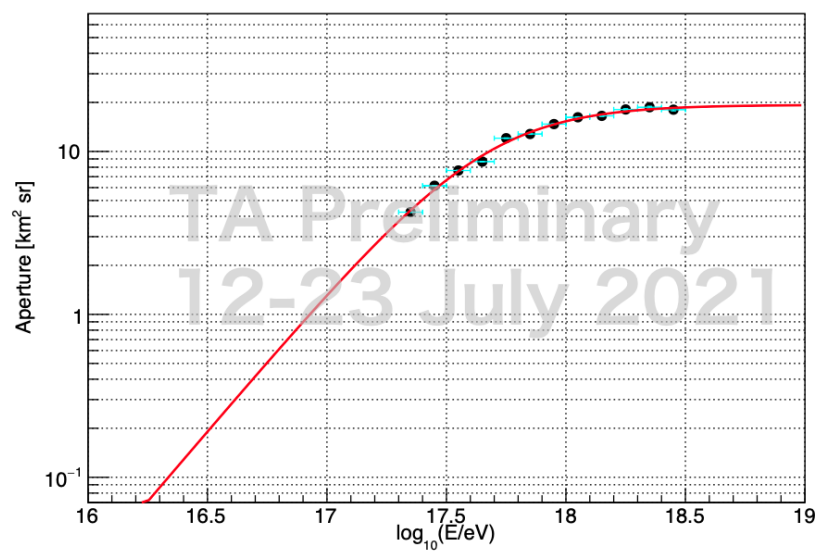

Figure 4: The aperture assuming a pure proton for the TALE-SD array. The red line is a fitted data point.

\section{Data/MC Comparisons}

To check if the Monte Carlo reasonably represents the data, measurable parameters' distributions were plotted. The Monte Carlo and Data (from October2019 to the end of January 2020) were applied the same quality cuts to be compared in energy range from $10^{17.5} \mathrm{eV}$ to $10^{18.5} \mathrm{eV}$. Figure 5 shows Data and Monte Carlo comparisons for primary energy E, azimuthal angle $\phi$, zenith angle $\theta$, the particle density at $600 \mathrm{~m}$ from the shower axis $\mathrm{S} 600$, core position in $\mathrm{X}$ and core position in Y. Blue data points and red Monte Carlo histogram are consistent. 

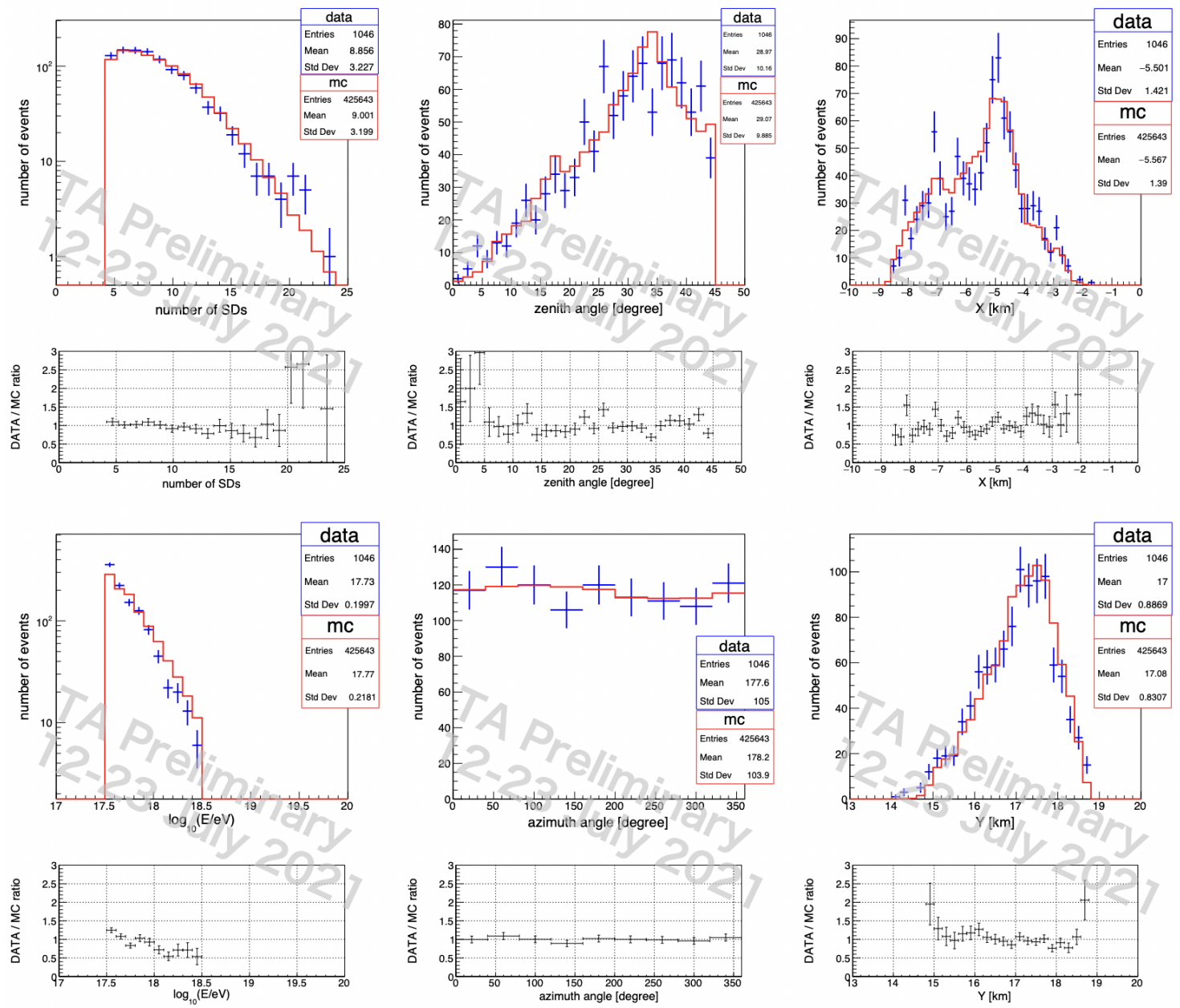

Figure 5: Data/Monte Carlo comparisons. Top left: the shower energy E. Top middle: the zenith angle, $\theta$. Top right: the azimuthal angle, $\phi$. Bottom left: the particle density at $600 \mathrm{~m}$ from the shower axis, S600. Bottom middle: the shower core position in $\mathrm{X}, \mathrm{x}$. Bottom right: the shower core position in $\mathrm{Y}$, y. points with error bars show the data, while the Monte Carlo is shown by the red histogram. The Monte Carlo has been normalized to the same number of events as the data.

\section{Conclusion}

The TALE SD array has been in operation since February 2018 with 80 SDs. The TALE-SD array DAQ is now running stably. We evaluate the energy resolutions of $20 \%$, in energy range from $10^{16.7} \mathrm{eV}$ to $10^{18.5} \mathrm{eV}$, and as for the resolution of the arrival direction, $3^{\circ}$ for events with energy $10^{16.7} \mathrm{eV}$ and $1.5^{\circ}$ for events with energy $10^{18.5} \mathrm{eV}$. And we evaluated the preliminary aperture assuming a pure proton. We also compared the MC and Data. There were no big differences. In the future, we plan to obtain the energy estimation table, the aperture by considering the composition of cosmic rays. 


\section{Acknowledgment}

The TALE SD production and the TALE hybrid operations are supported by the Japan Society for the Promotion of Science(JSPS) through Grants-in-Aid for Scientific Research (S) 15H05741 and 19H05607; by the joint research program of the Institute for Cosmic Ray Research (ICRR), The University of Tokyo. The experimental site became available through the cooperation of the Utah School and Institutional Trust Lands Administration (SITLA), U.S. Bureau of Land Management (BLM), and the U.S. Air Force. We appreciate the assistance of the State of Utah and Fillmore offices of the BLM in crafting the Plan of Development for the site. The people and the officials of Millard County, Utah have been a source of steadfast and warm support for our work which we greatly appreciate. We gratefully acknowledge the contribution from the technical staffs of our home institutions. The full acknowledgements are found in the contribution paper for the highlights of the Telescope Array experiments in this conference proceedings.

\section{References}

[1] S. Ogio, PoS ICRC2019 (2019) 375.

[2] G.Furlich, Ph.D. thesis, University of Utah, 2020.

[3] J. Linsley and L. Scarsi, Phys. Rev. 128 (1962) 2384.

[4] D. Ivanov, Ph.D. thesis, The state University of New Jersey, 2012.

[5] M. Teshima et al., J. Phys. G 12 (1986) 1097.

[6] S. Yoshida, N. Hayashida, K. Honda, M. Honda, S. Imaizumi, N. Inoue et al., Journal of Physics G: Nuclear and Particle Physics 20 (1994) 651.

[7] B.T. Stokes, R. Cady, D. Ivanov, J.N. Matthews and G.B. Thomson, Astropart. Phys. 35 (2012) 759 [1104.3182].

[8] S. Ostapchenko, Phys. Rev. D 83 (2011) 014018 [1010. 1869]. 


\section{Full Authors List: Telescope Array Collaboration}

R.U. Abbasi ${ }^{1}$, M. Abe ${ }^{2}$, T. Abu-Zayyad ${ }^{1,3}$, M. Allen $^{3}$, Y. Arai ${ }^{4}$, Y. Arimura ${ }^{4}$, E. Barcikowski ${ }^{3}$, J.W. Belz ${ }^{3}$, D.R. Bergman ${ }^{3}$, S.A. Blake ${ }^{3}$, I. Buckland ${ }^{3}$, R. Cady $^{3}$, B.G. Cheon ${ }^{5}$, J. Chiba ${ }^{6}$, M. Chikawa ${ }^{7}$, T. Fujii ${ }^{8}$, K. Fujisue ${ }^{7}$, K. Fujita ${ }^{4}$, R. Fujiwara ${ }^{4}$, M. Fukushima ${ }^{7,9}$, R. Fukushima ${ }^{4}$, G. Furlich ${ }^{3}$, R. Gonzalez ${ }^{3}$, W. Hanlon $^{3}$, M. Hayashi ${ }^{10}$, N. Hayashida ${ }^{11}$, K. Hibino ${ }^{11}$, R. Higuchi ${ }^{7}$, K. Honda ${ }^{12}$, D. Ikeda ${ }^{11}$, T. Inadomi ${ }^{13}$, N. Inoue ${ }^{2}$, T. Ishii ${ }^{12}$, H. Ito $^{14}$, D. Ivanov ${ }^{3}$, H. Iwakura ${ }^{13}$, A. Iwasaki ${ }^{4}$, H.M. Jeong ${ }^{15}$, S. Jeong ${ }^{15}$, C.C.H. Jui ${ }^{3}$, K. Kadota ${ }^{16}$, F. Kakimoto ${ }^{11}$, O. Kalashev ${ }^{17}$, K. Kasahara ${ }^{18}$, S. Kasami ${ }^{19}$, H. Kawai ${ }^{20}$, S. Kawakami ${ }^{4}$, S. Kawana ${ }^{2}$, K. Kawata ${ }^{7}$, E. Kido ${ }^{14}$, H.B. Kim ${ }^{5}$, J.H. Kim ${ }^{3}$, J.H. Kim ${ }^{3}$, M.H. Kim ${ }^{15}$, S.W. Kim ${ }^{15}$, Y. Kimura ${ }^{4}$, S. Kishigami ${ }^{4}$, Y. Kubota ${ }^{13}$, S. Kurisu ${ }^{13}$, V. Kuzmin ${ }^{17}$, M. Kuznetsov ${ }^{17,21}$, Y.J. Kwon ${ }^{22}$, K.H. Lee ${ }^{15}$, B. Lubsandorzhiev ${ }^{17}$, J.P. Lundquist ${ }^{3,23}$, K. Machida ${ }^{12}$, H. Matsumiya ${ }^{4}$, T. Matsuyama ${ }^{4}$, J.N. Matthews ${ }^{3}$, R. Mayta ${ }^{4}$, M. Minamino ${ }^{4}$, K. Mukai ${ }^{12}$, I. Myers ${ }^{3}$, S. Nagataki ${ }^{14}$, K. Nakai ${ }^{4}$, R. Nakamura ${ }^{13}$,

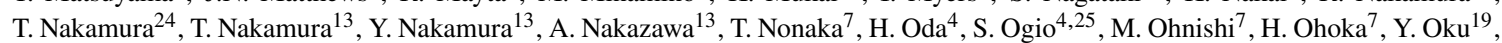
T. Okuda ${ }^{26}$, Y. Omura ${ }^{4}$, M. Ono ${ }^{14}$, R. Onogi ${ }^{4}$, A. Oshima ${ }^{4}$, S. Ozawa ${ }^{27}$, I.H. Park ${ }^{15}$, M. Potts ${ }^{3}$, M.S. Pshirkov ${ }^{17,28}$, J. Remington $^{3}$, D.C. Rodriguez ${ }^{3}$, G.I. Rubtsov ${ }^{17}$, D. Ryu ${ }^{29}$, H. Sagawa ${ }^{7}$, R. Sahara ${ }^{4}$, Y. Saito ${ }^{13}$, N. Sakaki ${ }^{7}$, T. Sako ${ }^{7}$, N. Sakurai ${ }^{4}$, K. Sano ${ }^{13}$, K. Sato ${ }^{4}$, T. Seki ${ }^{13}$, K. Sekino ${ }^{7}$, P.D. Shah ${ }^{3}$, Y. Shibasaki ${ }^{13}$, F. Shibata ${ }^{12}$, N. Shibata ${ }^{19}$, T. Shibata ${ }^{7}$, H. Shimodaira ${ }^{7}$, B.K. Shin ${ }^{29}$, H.S. Shin ${ }^{7}$, D. Shinto ${ }^{19}$, J.D. Smith ${ }^{3}$, P. Sokolsky ${ }^{3}$, N. Sone ${ }^{13}$, B.T. Stokes ${ }^{3}$, T.A. Stroman ${ }^{3}$, T. Suzawa ${ }^{2}$, Y. Takagi ${ }^{4}$, Y. Takahashi ${ }^{4}$, M. Takamura ${ }^{6}$,

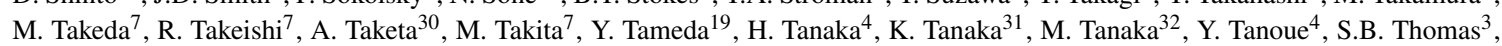
G.B. Thomson ${ }^{3}$, P. Tinyakov ${ }^{17,21}$, I. Tkachev ${ }^{17}$, H. Tokuno ${ }^{33}$, T. Tomida ${ }^{13}$, S. Troitsky ${ }^{17}$, R. Tsuda ${ }^{4}$, Y. Tsunesada ${ }^{4,25}$, Y. Uchihori ${ }^{34}$, S. Udo ${ }^{11}$, T. Uehama ${ }^{13}$, F. Urban ${ }^{35}$, T. Wong ${ }^{3}$, K. Yada ${ }^{7}$, M. Yamamoto ${ }^{13}$, K. Yamazaki ${ }^{11}$, J. Yang ${ }^{36}$, K. Yashiro ${ }^{6}$, F. Yoshida ${ }^{19}$, Y. Yoshioka ${ }^{13}$, Y. Zhezher ${ }^{7,17}$, and Z. Zundel ${ }^{3}$

${ }^{1}$ Department of Physics, Loyola University Chicago, Chicago, Illinois, USA

2 The Graduate School of Science and Engineering, Saitama University, Saitama, Saitama, Japan

${ }^{3}$ High Energy Astrophysics Institute and Department of Physics and Astronomy, University of Utah, Salt Lake City, Utah, USA

${ }^{4}$ Graduate School of Science, Osaka City University, Osaka, Osaka, Japan

${ }^{5}$ Department of Physics and The Research Institute of Natural Science, Hanyang University, Seongdong-gu, Seoul, Korea

${ }^{6}$ Department of Physics, Tokyo University of Science, Noda, Chiba, Japan

${ }^{7}$ Institute for Cosmic Ray Research, University of Tokyo, Kashiwa, Chiba, Japan

${ }^{8}$ The Hakubi Center for Advanced Research and Graduate School of Science, Kyoto University, Kitashirakawa-Oiwakecho, Sakyo-ku, Kyoto, Japan

${ }^{9}$ Kavli Institute for the Physics and Mathematics of the Universe (WPI), Todai Institutes for Advanced Study, University of Tokyo, Kashiwa, Chiba, Japan

${ }^{10}$ Information Engineering Graduate School of Science and Technology, Shinshu University, Nagano, Nagano, Japan

${ }^{11}$ Faculty of Engineering, Kanagawa University, Yokohama, Kanagawa, Japan

${ }^{12}$ Interdisciplinary Graduate School of Medicine and Engineering, University of Yamanashi, Kofu, Yamanashi, Japan

${ }_{13}$ Academic Assembly School of Science and Technology Institute of Engineering, Shinshu University, Nagano, Nagano, Japan

${ }^{14}$ Astrophysical Big Bang Laboratory, RIKEN, Wako, Saitama, Japan

${ }^{15}$ Department of Physics, Sungkyunkwan University, Jang-an-gu, Suwon, Korea

${ }^{16}$ Department of Physics, Tokyo City University, Setagaya-ku, Tokyo, Japan

${ }^{17}$ Institute for Nuclear Research of the Russian Academy of Sciences, Moscow, Russia

${ }^{18}$ Faculty of Systems Engineering and Science, Shibaura Institute of Technology, Minato-ku, Tokyo, Japan

${ }^{19}$ Department of Engineering Science, Faculty of Engineering, Osaka Electro-Communication University, Neyagawa-shi, Osaka, Japan

${ }^{20}$ Department of Physics, Chiba University, Chiba, Chiba, Japan

${ }^{21}$ Service de Physique Théorique, Université Libre de Bruxelles, Brussels, Belgium

${ }^{22}$ Department of Physics, Yonsei University, Seodaemun-gu, Seoul, Korea

${ }^{23}$ Center for Astrophysics and Cosmology, University of Nova Gorica, Nova Gorica, Slovenia

${ }^{24}$ Faculty of Science, Kochi University, Kochi, Kochi, Japan

${ }^{25}$ Nambu Yoichiro Institute of Theoretical and Experimental Physics, Osaka City University, Osaka, Osaka, Japan

${ }^{26}$ Department of Physical Sciences, Ritsumeikan University, Kusatsu, Shiga, Japan

${ }^{27}$ Quantum ICT Advanced Development Center, National Institute for Information and Communications Technology, Koganei, Tokyo, Japan

28 Sternberg Astronomical Institute, Moscow M.V. Lomonosov State University, Moscow, Russia

${ }^{29}$ Department of Physics, School of Natural Sciences, Ulsan National Institute of Science and Technology, UNIST-gil, Ulsan, Korea

${ }^{30}$ Earthquake Research Institute, University of Tokyo, Bunkyo-ku, Tokyo, Japan

${ }^{31}$ Graduate School of Information Sciences, Hiroshima City University, Hiroshima, Hiroshima, Japan

32 Institute of Particle and Nuclear Studies, KEK, Tsukuba, Ibaraki, Japan

33 Graduate School of Science and Engineering, Tokyo Institute of Technology, Meguro, Tokyo, Japan

34 Department of Research Planning and Promotion, Quantum Medical Science Directorate, National Institutes for Quantum and Radiological Science and Technology, Chiba, Chiba, Japan

35 CEICO, Institute of Physics, Czech Academy of Sciences, Prague, Czech Republic

${ }^{36}$ Department of Physics and Institute for the Early Universe, Ewha Womans University, Seodaaemun-gu, Seoul, Korea 